NORDITA-94/71 P

gr-qc/9502023

\title{
Square-Root Actions, Metric Signature, and the Path-Integral of Quantum Gravity
}

\author{
A. Carlini ${ }^{\diamond}$ and J. Greensite* \\ $\diamond_{\text {NORDITA, Blegdamsvej } 17}$ \\ DK-2100 Copenhagen $\varnothing$, Denmark \\ Email: carlini@nbivax.nbi.dk \\ *Physics and Astronomy Dept. \\ San Francisco State University \\ San Francisco, CA 94117, USA \\ Email: greensit@stars.sfsu.edu
}

\begin{abstract}
We consider quantization of the Baierlein-Sharp-Wheeler form of the gravitational action, in which the lapse function is determined from the Hamiltonian constraint. This action has a square root form, analogous to the actions of the relativistic particle and Nambu string. We argue that path-integral quantization of the gravitational action should be based on a path integrand $\exp [\sqrt{i} S]$ rather than the familiar Feynman expression $\exp [i S]$, and that unitarity requires integration over manifolds of both Euclidean and Lorentzian signature. We discuss the relation of this path integral to our previous considerations regarding the problem of time, and extend our approach to include fermions.
\end{abstract}




\section{Introduction}

Square-root Lagrangians are a feature of many field theories which are invariant under a time-reparametrization. The action of a relativistic particle

$$
S_{P}=-m \int d \tau \sqrt{-g_{\mu \nu} \partial_{\tau} x^{\mu} \partial_{\tau} x^{\nu}}
$$

and the action of the Nambu string

$$
S_{N}=T \int d \sigma d \tau \sqrt{\left(\partial_{\tau} \vec{x}\right)^{2}\left(\partial_{\sigma} \vec{x}\right)^{2}-\left(\partial_{\tau} \vec{x} \cdot \partial_{\sigma} \vec{x}\right)^{2}}
$$

are familiar examples. Somewhat less familiar is the Baierlein-Sharp-Wheeler (BSW) form of the gravitational action

$$
S_{B S W}=-\frac{1}{\kappa^{2}} \int d^{4} x \sqrt{\sqrt{g}^{3} R G^{i j n m}\left(\partial_{t} g_{i j}-2 N_{(i ; j)}\right)\left(\partial_{t} g_{n m}-2 N_{(n ; m)}\right)}
$$

which is obtained from the standard ADM action, as reviewed below, by solving the Hamiltonian constraint. It is well known that for a relativistic particle moving in an arbitrary curved background, and for gravity in general, the corresponding quantum theory lacks a well-defined probability measure and time-evolution parameter [1], [2], [3.

In this article we will propose a path-integral formulation of these "square-root" theories which is something of a departure from the standard Feynman expression. For one thing, the integrand of our path-integral will involve an unconventional phase:

$$
\exp [\sqrt{i} S] \quad \text { rather than } \exp [i S]
$$

Secondly, we will regularize the integration measure so as to uncover what we believe to be the true time-evolution parameter of the quantum theory. Third, we will find it necessary to sum over path-segments of both real and imaginary proper-time, i.e. over time-like and space-like trajectories in the case of the relativistic particle; Lorentzian and Euclidean signature manifolds in the case of gravity. It will be shown that the combination of the regularization, the unconventional phase, and the inclusion of imaginary proper-time segments, leads to a unitary evolution of states which corresponds, via the Ehrenfest principle, to the standard classical dynamics.

In two previous articles [4], [5] we have advocated a transfer-matrix approach to quantizing time reparametrization-invariant theories. The present article essentially presents the "real-time" version of our former "Euclidean" approach. Our previous work did not include fermion fields, which involve certain complications in our formulation. In this paper, we will show how the fermionic fields are also incorporated into our approach. 


\section{Minisuperspace Actions}

We begin by considering simple quantum-mechanical theories with a time-reparametrization invariance, i.e. the "minisuperspace" models of the form

$$
\begin{aligned}
S & =\int d t\left\{p_{a} \partial_{t} q^{a}-N H[p, q]\right\} \\
H & =\frac{1}{2 m} G^{a b}(q) p_{a} p_{b}+m V(q)
\end{aligned}
$$

where it is assumed that the supermetric $G^{a b}$ has Lorentzian signature $(-+++\ldots+)$. The "square-root" form of the action is obtained by solving for $p_{a}$ in terms of the time-derivatives of the $\left\{q^{a}\right\}$, i.e.

$$
\begin{aligned}
\partial_{t} q^{a} & =N \frac{\partial H}{\partial p_{a}}=\frac{N G^{a b} p_{b}}{m} \\
\Longrightarrow & p_{a}=\frac{m}{N} G_{a b} \partial_{t} q^{b}
\end{aligned}
$$

and then solving the Hamiltonian constraint for the lapse function

$$
\begin{aligned}
0 & =\frac{1}{2 m} G^{a b}(q) p_{a} p_{b}+m V(q) \\
& =\frac{m}{2 N^{2}} G_{a b} \partial_{t} q^{a} \partial_{t} q^{b}+m V(q) \\
\Longrightarrow N & =\sqrt{-\frac{G_{a b}}{2 V} \partial_{t} q^{a} \partial_{t} q^{b}}
\end{aligned}
$$

Substituting (6) and (7) into the minisuperspace action then gives the square-root form

$$
S=-m \int d t \sqrt{-2 V G_{a b} \partial_{t} q^{a} \partial_{t} q^{b}}
$$

For $V=\frac{1}{2}$, this is simply the action for a relativistic particle of mass $m$, moving in a background manifold with metric $G_{a b}$.

In non-relativistic quantum mechanics, a path-integral is constructed out of elementary integrals which evolve the wavefunction by a small time-interval $\epsilon$, i.e.

$$
\begin{aligned}
\psi\left(x^{\prime}, t+\epsilon\right) & =\int d^{D} x \mu_{\epsilon} \exp \left[i S\left[\left(x^{\prime}, t+\epsilon\right) ;(x, t)\right] / \hbar\right] \psi(x, t) \\
& =U_{\epsilon} \psi\left(x^{\prime}, t\right)
\end{aligned}
$$

where $S\left[\left(x^{\prime}, t+\epsilon\right) ;(x, t)\right]$ is the action of a classical trajectory between the points $x$ at time $t$ and $x^{\prime}$ at time $t+\epsilon$. The measure $\mu_{\epsilon}$ is chosen so that $\psi(x, t+\epsilon) \rightarrow \psi(x, t)$ as $\epsilon \rightarrow 0$. With this rule, one finds

$$
U_{\epsilon}=\exp [-i H \epsilon / \hbar]+O\left(\epsilon^{2}\right)
$$


where $H$ (the Hamiltonian) is an $\epsilon$-independent Hermitian operator. Taking the $\epsilon \rightarrow 0$ limit, the evolution operator for finite times

$$
\begin{aligned}
U_{\Delta t} & \equiv \lim _{\epsilon \rightarrow 0}\left(U_{\epsilon}\right)^{\Delta t / \epsilon} \\
& =\exp [-i H \Delta t / \hbar]
\end{aligned}
$$

is a unitary operator. Straightforward imitation of this construction doesn't work in the case of the square-root theories, due to the time-reparametrization invariance. Because of this invariance, the action of a classical trajectory between an initial point $q$ and an end point $q^{\prime}$ is independent of the time parameters $t$ and $t+\epsilon$ which label those configurations, i.e.

$$
S\left[\left(q^{\prime}, t+\epsilon\right) ;(q, t)\right]=S\left[q^{\prime}, q\right]
$$

The resulting operator $U_{\epsilon}$ defined from (9) would therefore be $\epsilon$-independent, and also in general non-unitary.

Let us see if it is possible to recover an evolution operator of the form (10) for the square-root actions, by making a slight change to the construction shown in eq. (9). The modification is to multiply the action $S\left[q^{\prime}, q\right]$ by an $\epsilon$-dependent complex constant $c_{\epsilon}$

$$
\begin{aligned}
\psi\left(q^{\prime}, \tau+\epsilon\right) & =\int d^{D} q \mu_{\epsilon} \exp \left[c_{\epsilon} S\left(q^{\prime}, q\right)\right] \psi(q, \tau) \\
& =U_{\epsilon} \psi\left(q^{\prime}, \tau\right)
\end{aligned}
$$

which is to be chosen such that $U_{\epsilon}$ is a unitary operator (up to order $\epsilon$ ) of the form

$$
U_{\epsilon}=\exp [-i Æ \epsilon / \hbar]+O\left(\epsilon^{2}\right)
$$

where $\rightleftarrows$ is an $\epsilon$-independent operator, hermitian in the measure $\mu_{\epsilon}$. Begin, for simplicity, with a "minisuperspace" action having $V=\frac{1}{2}$ and $G_{a b}=\eta_{a b}$; i.e. the action of a relativistic particle in flat D-dimensional Minkowski space. Let

$$
x^{\prime \mu}=x^{\mu}-\Delta x^{\mu}
$$

so that

$$
\psi\left(x^{\prime}, \tau+\epsilon\right)=\int d^{D} x \mu_{\epsilon} \exp \left[-c_{\epsilon} m \sqrt{-\eta_{a b} \Delta x^{a} \Delta x^{b}}\right] \psi(x, \tau)
$$

Comparing this expression to the corresponding expression for a free non-relativistic particle

$$
\psi\left(x^{\prime}, \tau+\epsilon\right)=\int d^{D} x \mu_{\epsilon} \exp \left[m \frac{\delta_{i j} \Delta x^{i} \Delta x^{j}}{(-i \epsilon \hbar)}\right] \psi(x, t)
$$


motivates us to try

$$
c_{\epsilon}=\frac{1}{\sqrt{-i \epsilon \hbar}}
$$

with the understanding that the "time"-step $\epsilon$ now has units of action, and that the branch of the square-root is chosen so that the exponent in (16) has a negative real part. This choice does not quite complete the definition of $U_{\epsilon}$, as there is still a question of the range of the integral over $x$. Should this integral range over all possible $x$, or should it be restricted so that the path-segment $\Delta x^{a}$ is timelike? To resolve this issue, we will compute separately the contributions from timelike and spacelike intervals.

Following the usual steps leading from the path integral to the Schrödinger equation, expand $\psi(x, \tau)$ in a Taylor series around $x^{\prime}$

$$
\begin{aligned}
\psi\left(x^{\prime}, \tau+\epsilon\right)= & \int d^{D} x \mu_{\epsilon} \exp \left[-m \sqrt{-\eta_{\mu \nu} \Delta x^{\nu} \Delta x^{\mu}} / \sqrt{-i \epsilon \hbar}\right] \\
& \times\left[\psi\left(x^{\prime}, \tau\right)+\frac{\partial \psi}{\partial x^{\prime \mu}} \Delta x^{\nu}+\frac{1}{2} \frac{\partial^{2} \psi}{\partial x^{\prime \mu} \partial x^{\prime \nu}} \Delta x^{\mu} \Delta x^{\nu}+\ldots\right] \\
= & U_{\epsilon} \psi\left(x^{\prime}, t\right)
\end{aligned}
$$

In order that

$$
\lim _{\epsilon \rightarrow 0} U_{\epsilon}=1
$$

the measure must be chosen to be

$$
\mu_{\epsilon}^{-1}=\int d^{D} x \exp \left[-m \sqrt{-\eta_{\mu \nu} \Delta x^{\mu} \Delta x^{\nu}} / \sqrt{-i \epsilon \hbar}\right]
$$

Changing variables $\Delta x \rightarrow x$, we then have

$$
U_{\epsilon}=1+\frac{1}{2}\left[\int d^{D} x \mu x^{\mu} x^{\nu} \exp \left[-m \sqrt{-\eta_{\mu \nu} x^{\mu} x^{\nu}} / \sqrt{-i \epsilon \hbar}\right]\right] \partial_{\mu} \partial_{\nu}+\ldots
$$

Denote $x^{\mu}=\{t, \vec{x}\}$ and $r^{2}=\vec{x} \cdot \vec{x}$. Then, on grounds of relativistic covariance,

$$
\begin{aligned}
U_{\epsilon} & =1+\frac{1}{2}\left[\int d^{D} x \mu \frac{r^{2} \eta^{\mu \nu}}{(D-1)} \exp \left[-m \sqrt{-\eta_{\mu \nu} x^{\mu} x^{\nu}} / \sqrt{-i \epsilon \hbar}\right]\right] \partial_{\mu} \partial_{\nu}+\ldots \\
& =1+\frac{1}{2(D-1)} \frac{I_{B}}{I_{A}} \partial^{2}+\ldots
\end{aligned}
$$

where $\partial^{2}=\eta^{\mu \nu} \partial_{\mu} \partial_{\nu}$, and

$$
\begin{aligned}
I_{A} & \equiv \int d^{D} x \exp \left[-m \sqrt{-\eta_{\mu \nu} x^{\mu} x^{\nu}} / \sqrt{-i \epsilon \hbar}\right] \\
I_{B} & \equiv \int d^{D} x r^{2} \exp \left[-m \sqrt{-\eta_{\mu \nu} x^{\mu} x^{\nu}} / \sqrt{-i \epsilon \hbar}\right]
\end{aligned}
$$

We first evalute $I_{A}$; the second integral $I_{B}$ will follow easily. Starting with

$$
I_{A}=\sigma \int d t d r r^{D-2} \exp \left[-m \sqrt{t^{2}-r^{2}} / \sqrt{-i \epsilon \hbar}\right]
$$


where

$$
\sigma=\frac{2 \pi^{(D-1) / 2}}{\Gamma\left(\frac{D-1}{2}\right)}
$$

divide the integral over $t$ into two contributions, one from timelike and one from spacelike paths:

$$
\begin{aligned}
I_{A}= & \sigma \int_{0}^{\infty} d r r^{D-2}\left\{\int_{r}^{\infty} d t \exp \left[-m \sqrt{t^{2}-r^{2}} / \sqrt{-i \epsilon \hbar}\right]\right. \\
& \left.+\int_{0}^{r} d t \exp \left[-m \sqrt{r^{2}-t^{2}} / \sqrt{i \epsilon \hbar}\right]\right\}
\end{aligned}
$$

where the branches of the square roots $\sqrt{-i \epsilon \hbar}$ and $\sqrt{i \epsilon \hbar}$ are taken with positive real parts, to ensure convergence of the integrals. Next

$$
\begin{aligned}
I_{A}= & \sigma \int_{0}^{\infty} d r r^{D-2}\left\{\int_{r}^{\infty} d t \exp \left[-m \sqrt{t^{2}-r^{2}} / \sqrt{-i \epsilon \hbar}\right] \int_{0}^{\infty} d y 2 y \delta\left[y^{2}-\left(t^{2}-r^{2}\right)\right]\right. \\
& \left.+\int_{0}^{r} d t \exp \left[-m \sqrt{r^{2}-t^{2}} / \sqrt{i \epsilon \hbar}\right] \int_{0}^{\infty} d y 2 y \delta\left[y^{2}-\left(r^{2}-t^{2}\right)\right]\right\} \\
= & \sigma \int_{0}^{\infty} d r r^{D-2} \int_{0}^{\infty} d y\left\{\frac{y}{\sqrt{y^{2}+r^{2}}} e^{-m y / \sqrt{-i \epsilon \hbar}}+\frac{y}{\sqrt{r^{2}-y^{2}}} e^{-m y / \sqrt{i \epsilon \hbar}} \Theta(r-y)\right\} \\
= & \sigma \int_{0}^{\infty} d r r^{D-2}\left\{F_{1}(r)+F_{2}(r)\right\}
\end{aligned}
$$

where

$$
\begin{aligned}
& F_{1}(r) \equiv \int_{0}^{\infty} d y \frac{y}{\sqrt{y^{2}+r^{2}}} e^{-m y / \sqrt{-i \epsilon \hbar}} \\
& F_{2}(r) \equiv \int_{0}^{r} d y \frac{y}{\sqrt{r^{2}-y^{2}}} e^{-m y / \sqrt{i \epsilon \hbar}}
\end{aligned}
$$

It is easy to see that asymptotically, as $r \rightarrow \infty$, both $F_{1}(r)$ and $F_{2}(r)$ go like $1 / r$. But that implies

$$
\begin{aligned}
\text { timelike paths contribution }=\sigma \int_{0}^{\infty} d r r^{D-2} F_{1}(r) & \text { is divergent } \\
\text { spacelike paths contribution }=\sigma \int_{0}^{\infty} d r r^{D-2} F_{2}(r) & \text { is divergent }
\end{aligned}
$$

This means that if we were to restrict the paths to only timelike, or only spacelike paths, then $I_{A}$ (and $I_{B}$ ) would be hopelessly divergent, and the evolution operator $U_{\epsilon}$ would be ill-defined. The remarkable thing, which we now show, is that the sum of the two contributions is actually finite.

Let us deform the contour of $y$-integration for the integral defining $F_{1}(r)$ in eq. (29). As it stands, it runs along the real axis from 0 to $\infty$. Deform it to run along the imaginary axis from 0 to $-i r$, and then parallel to the real axis from $-i r$ to $\infty$. There are no poles or branch cuts in the way, so the deformation is permissible. Then 


$$
F_{1}(r)=\left\{\int_{0}^{-i r} d y+\int_{-i r}^{\infty} d y\right\} \frac{y}{\sqrt{r^{2}+y^{2}}} e^{-m y / \sqrt{-i \epsilon \hbar}}
$$

Change variables, $y \rightarrow-i y$

$$
F_{1}(r)=-\int_{0}^{r} d y \frac{y}{\sqrt{r^{2}-y^{2}}} e^{-m y / \sqrt{i \epsilon \hbar}}-\int_{r}^{i \infty} d y \frac{y}{\sqrt{r^{2}-y^{2}}} e^{-m y / \sqrt{i \epsilon \hbar}}
$$

Its not hard to see that it is the first integral which causes the divergence of the $r$-integration. Adding together $F_{1}$ and $F_{2}$, observe that the first integral in (32) exactly cancels $F_{2}$, leaving an expression which decays exponentially as $r$ increases

$$
F_{1}+F_{2}=-\int_{r}^{i \infty} d y \frac{y}{\sqrt{r^{2}-y^{2}}} e^{-m y / \sqrt{i \epsilon \hbar}}
$$

The contour of this integral runs parallel to the (positive) imaginary axis. Now rotate the contour by 90 degrees, so that it runs along the positive real axis. Again, there are no poles or branch cuts in the way, and the integral is convergent along any contour intermediate between the initial contour, and the 90 degree rotated contour. This gives

$$
\begin{aligned}
F_{1}+F_{2} & =i \int_{r}^{\infty} d y \frac{y}{\sqrt{y^{2}-r^{2}}} e^{-m y / \sqrt{i \epsilon \hbar}} \\
& =i r K_{1}\left(\frac{m r}{\sqrt{i \epsilon \hbar}}\right)
\end{aligned}
$$

Inserting this result into (28), one finds

$$
\begin{aligned}
I_{A} & =i \sigma \int_{0}^{\infty} d r r^{D-1} K_{1}\left(\frac{m r}{\sqrt{i \epsilon \hbar}}\right) \\
& =i \sigma 2^{D-2}\left(\frac{\sqrt{i \epsilon \hbar}}{m}\right)^{D} \Gamma\left(\frac{D+1}{2}\right) \Gamma\left(\frac{D-1}{2}\right)
\end{aligned}
$$

It is trivial to repeat all the above steps for the $I_{B}$ integral, and the result is

$$
\begin{aligned}
I_{B} & =i \sigma \int_{0}^{\infty} d r r^{D+1} K_{1}\left(\frac{m r}{\sqrt{i \epsilon \hbar}}\right) \\
& =i \sigma 2^{D}\left(\frac{\sqrt{i \epsilon \hbar}}{m}\right)^{D+2} \Gamma\left(\frac{D+1}{2}+1\right) \Gamma\left(\frac{D-1}{2}+1\right)
\end{aligned}
$$

Finally, inserting (35) and (36) into the expression for the evolution operator, eq. (23), we obtain

$$
\begin{aligned}
U_{\epsilon} & =1+i \epsilon \hbar \frac{(D+1)}{2 m^{2}} \eta^{\mu \nu} \partial_{\mu} \partial_{\nu}+O\left(\epsilon^{2}\right) \\
& =\exp [-i \notin \epsilon / \hbar]+O\left(\epsilon^{2}\right)
\end{aligned}
$$

where

$$
\rightleftarrows=-\frac{\hbar^{2}}{2 m^{2}}(D+1) \eta^{\mu \nu} \partial_{\mu} \partial_{\nu}
$$


The operator $\rightleftarrows$ is $\epsilon$-independent, and clearly Hermitian for inner products

$$
<\psi_{1}|Q| \psi_{2}>=\int d^{D} x \mu_{\epsilon} \psi_{1}^{*}(x, \tau) Q \psi_{2}(x, \tau)
$$

The evolution operator

$$
\begin{aligned}
U_{\Delta \tau} & \equiv \lim _{\epsilon \rightarrow 0}\left(U_{\epsilon}\right)^{\Delta \tau / \epsilon} \\
& =\exp [-i \circledast \Delta \tau / \hbar]
\end{aligned}
$$

is therefore unitary, as in the usual path-integral approach for theories without a time-reparametrization invariance.

It should be emphasized that the unitarity of our proposed evolution operator depends both on the choice of complex constant $c_{\epsilon}=1 / \sqrt{-i \epsilon \hbar}$, and also on summation over both timelike and spacelike path segments. A glance at equations (35) and (36) shows that the crucial factor of $i \epsilon$ in (37) could only be obtained if a $1 / \sqrt{-i \epsilon}$ factor multiplies the action in (13). Furthermore, the finiteness of the result depends on keeping contributions to the integrand from both timelike and spacelike path-segments; the integral over either contribution separately is divergent.

It is easy to generalize from the relativistic particle action to any minisuperspace square-root action of the form (8). First define the modified supermetric

$$
\mathcal{G}_{a b} \equiv 2 V G_{a b}
$$

For $\Delta q^{a}$ small

$$
\begin{aligned}
S\left[q^{\prime}, q\right] & =-m \int_{0}^{\Delta t} d t \sqrt{-\mathcal{G}_{a b} \partial_{t} q^{a} \partial_{t} q^{b}} \\
& =-m \sqrt{-\mathcal{G}_{a b} \Delta q^{a} \Delta q^{b}}
\end{aligned}
$$

The measure is

$$
\mu_{\epsilon}^{-1}\left(q^{\prime}\right)=(\sqrt{\epsilon \hbar})^{D} \lim _{\epsilon \rightarrow 0} \int \frac{d^{D} q}{(\sqrt{\epsilon \hbar})^{D}} \exp \left[-m \sqrt{-\mathcal{G}_{a b} \Delta q^{a} \Delta q^{b}} / \sqrt{-i \epsilon \hbar}\right]
$$

Now introduce Riemann normal coordinates $\xi^{a}$ around the point $q^{\prime a}$, which transforms the modified supermetric into the Minkowski metric $\mathcal{G}_{a b}=\eta_{a b}$ at the point $q^{\prime}$ $(\xi=0)$. Then

$$
\begin{aligned}
\mu_{\epsilon}^{-1}\left(q^{\prime}\right) & =\int d^{D} \xi\left|\operatorname{det}\left[\frac{\partial \Delta q^{a}}{\partial \xi^{b}}\right]\right| \exp \left[-m \sqrt{-\eta_{\alpha \beta} \xi^{a} \xi^{b}} / \sqrt{-i \epsilon \hbar}\right] \\
& =\frac{1}{\sqrt{\left|\mathcal{G}\left(q^{\prime}\right)\right|}} I_{A}
\end{aligned}
$$


Inserting this measure, and $c_{\epsilon}=1 / \sqrt{-i \epsilon \hbar}$, into eq. (13), we have

$$
\begin{aligned}
\psi\left(q^{\prime}, \tau+\epsilon\right)= & \frac{1}{I_{A}} \int d^{D} \Delta q \sqrt{\left|\mathcal{G}\left(q^{\prime}+\Delta q\right)\right|} \exp \left[-m \sqrt{-\mathcal{G}_{a b} \Delta q^{a} \Delta q^{b}} / \sqrt{-i \epsilon \hbar}\right] \\
& \times \psi\left(q^{\prime}+\Delta q, \tau\right) \\
= & \frac{1}{I_{A}} \int d^{D} \xi\left(1-\frac{1}{6} \mathcal{R}_{a b} \xi^{a} \xi^{b}+\ldots\right) \exp \left[-m \sqrt{-\eta_{a b} \xi^{a} \xi^{b}} / \sqrt{-i \epsilon \hbar}\right] \\
& \times\left\{\psi\left(q^{\prime}, \tau\right)+\frac{\partial \psi}{\partial \xi^{c}} \xi^{c}+\frac{1}{2} \frac{\partial^{2} \psi}{\partial \xi^{c} \partial \xi^{d}} \xi^{c} \xi^{d}+O\left(\xi^{3}\right)\right\} \\
= & {\left[1+\frac{1}{(D-1)} \frac{I_{B}}{I_{A}}\left\{\frac{1}{2} \partial^{2}-\frac{1}{6} \mathcal{R}+O\left(\epsilon^{2}\right)\right\}\right] \psi\left(q^{\prime}(\xi), \tau\right) } \\
= & {\left[1+i \epsilon \hbar \frac{(D+1)}{2 m^{2}} \eta^{a b} \frac{\partial^{2}}{\partial \xi^{a} \partial \xi^{b}}-i \epsilon \hbar \frac{(D+1)}{6 m^{2}} \mathcal{R}\right] \psi(\xi, \tau) }
\end{aligned}
$$

where $\mathcal{R}$ is the curvature scalar formed from the metric $\mathcal{G}_{a b}$. Transforming back from Riemann normal coordinates, we have

$$
\begin{aligned}
\psi(q, \tau+\epsilon) & =\left[1+i \epsilon \hbar \frac{(D+1)}{2 m^{2}} \frac{1}{\sqrt{|\mathcal{G}|}} \frac{\partial}{\partial q^{a}} \sqrt{|\mathcal{G}|} \mathcal{G}^{a b} \frac{\partial}{\partial q^{b}}-i \epsilon \hbar \frac{(D+1)}{6 m^{2}} \mathcal{R}\right] \psi(q, \tau) \\
& =\left[\exp [-i Æ \epsilon / \hbar]+O\left(\epsilon^{2}\right)\right] \psi(q, \tau) \\
& =U_{\epsilon} \psi(q, \tau)
\end{aligned}
$$

As in the relativistic particle case, $\exp [-i Æ \epsilon / \hbar]$ is a unitary operator, where

$$
Æ=-\hbar^{2} \frac{(D+1)}{2 m^{2}} \frac{1}{\sqrt{|\mathcal{G}|}} \frac{\partial}{\partial q^{a}} \sqrt{|\mathcal{G}|} \mathcal{G}^{a b} \frac{\partial}{\partial q^{b}}+\hbar^{2} \frac{(D+1)}{6 m^{2}} \mathcal{R}
$$

is obviously Hermitian in the measure $\mu_{\epsilon}$ of eq. (44). Taking the $\epsilon \rightarrow 0$ limit, the wavefunction $\psi(q, \tau)$ satisfies a Schrödinger equation

$$
i \hbar \partial_{\tau} \psi(q, \tau)=Æ \psi(q, \tau)
$$

The Schrödinger evolution equations (47) and (48) for time reparametrization invariant theories have been obtained by us previously, in refs. [4] and [5], from a transfer matrix approach. The transfer matrix involves integration over a purely real integrand, but in our case the cost was not simply a Wick rotation of the evolution parameter $\tau$, but also a rather unnatural rotation of signature of the modified supermetric $\mathcal{G}_{a b}$ from Lorentzian to Euclidean. This rotation then had to be undone in postulating the Schrödinger equation (48). We have now seen that this supermetric signature rotation can be avoided, and unitary Schrödinger evolution is derived directly. In refs. [4] and [5] the correspondence of this evolution to the usual classical dynamics was also discussed. In the interest of completeness we will 
briefly review this correspondence here, and refer the reader to the cited references for further details.

The classical quantity $Æ_{c l}$ corresponding to the operator $Æ$ is obtained by replacing derivatives with c-number momenta

$$
\rightleftarrows_{c l}\left[q^{a}, p_{a}\right]=\lim _{\hbar \rightarrow 0} \circledast\left[q^{a},-i \hbar \frac{\partial}{\partial q^{a}} \rightarrow p_{a}\right]
$$

which gives

$$
Æ_{c l}=(D+1) \frac{\frac{1}{2 m} G^{a b} p_{a} p_{b}}{m V}
$$

The Poisson bracket evolution equation

$$
\partial_{\tau} Q=\left\{Q, \mathbb{E}_{c l}\right\} \quad, \quad \mathbb{E}_{c l}=-\mathcal{E}
$$

is easily checked to be equivalent, up to a time-reparametrization, to the standard brackets

$$
\partial_{t} Q=\left\{Q, N \mathcal{H}^{\mathcal{E}}\right\} \quad, \quad \mathcal{H}^{\mathcal{E}}=0
$$

where

$$
\mathcal{H}^{\mathcal{E}}=\frac{1}{2 m \sqrt{\mathcal{E}}} G^{a b} p_{a} p_{b}+\sqrt{\mathcal{E}} m V
$$

The parameter $\mathcal{E}$ is classically irrelevant, in the sense that it drops out of the EulerLagrange equations; the square-root action corresponding to $\mathcal{H}^{\mathcal{E}}$ is

$$
S^{\mathcal{E}}=-\sqrt{\mathcal{E}} m \int d t \sqrt{-2 V G_{a b} \partial_{t} q^{a} \partial_{t} q^{b}}
$$

Since $\mathcal{E}$ only appears as a parameter multiplying the action, the fact that it drops out of the Euler-Lagrange equations is obvious. The same can be said for the mass of a relativistic particle in free-fall, the tension of the Nambu string, or Newton's constant in pure gravity. None of these parameters appears in the equations of motion at the classical level.

Because of the classical equivalence of the Poisson bracket equations (51) and (52), it is clear that the Schrödinger evolution (48) will obey an appropriate Ehrenfest principle, with certain quantum corrections due to the measure. The general solution $\psi(q, \tau)$ can be expanded in terms of stationary states

$$
\psi(q, \tau)=\sum_{\mathcal{E}} \phi_{\mathcal{E}}(q) e^{i \mathcal{E} \tau / \hbar}
$$

where

$$
Æ \phi_{\mathcal{E}}=-\mathcal{E} \phi_{\mathcal{E}}
$$


This $\tau$-independent equation can be rewritten in the form

$$
\left[-\frac{\hbar^{2}}{\mathcal{E}} \frac{(D+1)}{4 m^{2}} \frac{V}{\sqrt{|\mathcal{G}|}} \frac{\partial}{\partial q^{a}} V^{-1} \sqrt{|\mathcal{G}|} G^{a b} \frac{\partial}{\partial q^{b}}+\frac{\hbar^{2}}{\mathcal{E}} \frac{(D+1)}{6 m^{2}} V \mathcal{R}+V\right] \phi_{\mathcal{E}}(q)=0
$$

which is a Wheeler-DeWitt equation with a particular choice of operator-ordering and a (classically irrelevant) parameter $\mathcal{E}$, which can be absorbed into a redefinition of either $m$ or $\hbar$.

In the standard Dirac canonical quantization of actions of the form (5), the physical states must satisy a Wheeler-DeWitt equation of the form

$$
\begin{aligned}
\mathcal{H} \phi(q) & =\left[\frac{-\hbar^{2}}{2 m} " G^{a b} \frac{\partial^{2}}{\partial q^{a} \partial q^{b}} "+m V\right] \phi(q) \\
& =0
\end{aligned}
$$

where the quotation marks indicate an operator-ordering ambiguity. However, multiplying the action by an arbitrary constant $\sqrt{\mathcal{E}}$ converts this constraint to

$$
\begin{aligned}
\mathcal{H}^{\mathcal{E}} \phi(q) & =\left[\frac{-\hbar^{2}}{2 m \sqrt{\mathcal{E}}} " G^{a b} \frac{\partial^{2}}{\partial q^{a} \partial q^{b}} "+\sqrt{\mathcal{E}} m V\right] \phi(q) \\
& =0
\end{aligned}
$$

Because $\mathcal{E}$ is irrelevant at the classical level, there is no overriding reason that it should be a fixed parameter at the quantum level. In essence, our approach enlarges the space of physical states as the Hilbert space spanned by all states satisfying

$$
\mathcal{H}^{\mathcal{E}} \phi_{\mathcal{E}}(q)=0
$$

and it is this enlargement of the space of states which enables us to obtain nonstationary states $\psi(q, \tau)$. Moreover, our approach fixes the operator-ordering, as seen in eq. (57), at least for the minisuperspace theories. Further discussion of these points may be found in ref. [5].

Returning to eq. (13), the path integral for square-root actions is now defined as the limit

$$
\begin{aligned}
\psi\left(q_{f}, \tau_{0}+\Delta \tau\right) & =\int D q\left(\tau_{0} \leq \tau<\tau_{0}+\Delta \tau\right) e^{c_{0} S[q(\tau)]} \psi\left(q_{0}, \tau_{0}\right) \\
& =\lim _{\epsilon \rightarrow 0} \int \prod_{n=0}^{\Delta \tau / \epsilon-1} d^{D} q_{n} \mu_{\epsilon}\left(q_{n}\right) \exp \left[\frac{1}{\sqrt{-i \epsilon \hbar}} \sum_{m=0}^{\Delta \tau / \epsilon-1} S\left[q_{m+1}, q_{m}\right]\right] \psi\left(q_{0}, \tau_{0}\right) \\
& =\lim _{\epsilon \rightarrow 0}\left(U_{\epsilon}\right)^{\Delta \tau / \epsilon} \psi\left(q_{f}, \tau_{0}\right)
\end{aligned}
$$

where $\Delta \tau=\tau_{f}-\tau_{0}$. We see that the time parameter emerges from a regularization of the path-integral measure: at fixed $\epsilon$, a regularized path between the initial point 
$q_{0}$ and the final point $q_{f}$ consists of $n_{p}=\Delta \tau / \epsilon$ path segments, each segment being a classical trajectory between intermediate points $q_{n}$ and $q_{n+1}$. The evolution parameter is therefore a measure of the number of independent configurations (points) $n_{p}$ in the path joining $q_{0}$ to $q_{f}$, multiplied by the regularization interval, i.e.

$$
\Delta \tau=n_{p} \epsilon
$$

This is a quantum-mechanical time variable with no direct connection to, e.g., the proper time lapse. Nor is it an "intrinsic" time variable; all dynamical degrees of freedom are treated on the same footing and none is singled out as an evolution variable. Our evolution parameter is here identified as proportional to the number of "quantum steps" taken by the system in evolving from the initial to the final configuration. In this formulation the Green's functions are transitive, and the evolution of states is unitary.

\section{The BSW Action}

As in the minisuperspace case, the square-root form of the full gravitational action is derived from the first-order ADM action by solving for the lapse function. The $\mathrm{ADM}$ action for pure gravity is

$$
\begin{aligned}
S & =\int d^{4} x\left[p^{i j} \partial_{t} g_{i j}-N \mathcal{H}-N_{i} \mathcal{H}^{i}\right] \\
\mathcal{H} & =\kappa^{2} G_{i j k l} p^{i j} p^{k l}-\frac{1}{\kappa^{2}} \sqrt{g}{ }^{3} R \\
\mathcal{H}^{i} & =-2 p_{; k}^{i k} \\
G_{i j k l} & =\frac{1}{2 \sqrt{g}}\left(g_{i k} g_{j l}+g_{i l} g_{j k}-g_{i j} g_{k l}\right)
\end{aligned}
$$

where $g_{i j}$ is the metric of a 3 -manifold and ${ }^{3} R$ is the corresponding scalar curvature. The momentum is related to the time-derivative of the metric by

$$
\begin{aligned}
\partial_{t} g_{i j} & =N \frac{\partial \mathcal{H}}{\partial p^{i j}} \\
& =2 \kappa^{2} N G_{i j k l} p^{k l}+N_{i ; j}+N_{j ; i} \\
\Longrightarrow p^{i j} & =\frac{1}{2 \kappa^{2} N} G^{i j k l}\left(\partial_{t} g_{k l}-2 N_{(k ; l)}\right)
\end{aligned}
$$

Solving the Hamiltonian constraint for the lapse function

$$
\begin{aligned}
0 & =\frac{1}{4 \kappa^{2} N^{2}} G^{i j k l}\left(\partial_{t} g_{i j}-2 N_{(i ; j)}\right)\left(\partial_{t} g_{k l}-2 N_{(k ; l)}\right)-\frac{1}{\kappa^{2}} \sqrt{g}{ }^{3} R \\
\Longrightarrow N & =\left[\frac{1}{4 \sqrt{g}^{3} R} G^{i j k l}\left(\partial_{t} g_{i j}-2 N_{(i ; j)}\right)\left(\partial_{t} g_{k l}-2 N_{(k ; l)}\right)\right]^{1 / 2}
\end{aligned}
$$


and replacing the momenta in (63) by the expression (64), with lapse (65), gives the Baierlein-Sharp-Wheeler action [6]

$$
S_{B S W}=-\frac{1}{\kappa^{2}} \int d^{4} x \sqrt{\sqrt{g}^{3} R G^{i j n m}\left(\partial_{t} g_{i j}-2 N_{(i ; j)}\right)\left(\partial_{t} g_{n m}-2 N_{(n ; m)}\right)}
$$

Before quantizing, it is convenient to fix the coordinate system by choosing shift functions $N_{i}=0$. Then the corresponding supermomentum contraints $\delta S / \delta N_{i}=$ $\mathcal{H}^{i}=0$ are to be imposed as operator constraints on the space of physical states.

It is straightforward to extend the BSW action to include non-gravitational bosonic fields. To compress indices somewhat, we introduce the notation

$$
\begin{aligned}
\{a=1-6\} & \leftrightarrow\{(i, j), i \leq j\} \\
q^{a}(x) & \leftrightarrow g_{i j}(x) \\
p_{a}(x) & \leftrightarrow\left\{\begin{array}{cc}
p^{i j}(x) & (i=j) \\
2 p^{i j}(x) & (i<j)
\end{array}\right. \\
G_{a b}(x) & \leftrightarrow G^{i j n m}(x)
\end{aligned}
$$

and the non-gravitational fields are represented by $q^{a}(x)$ with indices $a>6$. It is convenient to rescale all non-gravitational fields by an appropriate power of $\kappa$ so that all fields, and all components of the supermetric, are dimensionless. The action is

$$
\begin{aligned}
S & =\int d^{4} x\left[p_{a} \partial_{t} q^{a}-N \mathcal{H}-N_{i} \mathcal{H}^{i}\right] \\
\mathcal{H} & =\kappa^{2} G^{a b} p_{a} p_{b}+\sqrt{g} U
\end{aligned}
$$

where

$$
\sqrt{g} U=-\frac{1}{\kappa^{2}} \sqrt{g}^{3} R+\text { non-gravitational contributions }
$$

Setting the shift functions to zero and repeating the above steps of solving for the lapse, gives again a square-root action

$$
S=-\frac{1}{\kappa} \int d^{4} x \sqrt{-\sqrt{g} U G_{a b} \partial_{t} q^{a} \partial_{t} q^{b}}
$$

The next step is to construct the evolution operator $U_{\epsilon}$ for the BSW action in the path-integral approach, following the procedure of the last section. The evolution operator is defined by

$$
\begin{aligned}
& \Psi\left[q^{\prime}(x), \tau+\epsilon\right]=\int D q(x) \mu(q) e^{\Delta S / \sqrt{-i \epsilon \hbar}}\left[\Psi\left[q^{\prime}(x)\right]+\int d^{3} x\left(\frac{\delta \Psi}{\delta q^{a}(x)}\right) \Delta q^{a}(x)\right. \\
& \left.+\frac{1}{2} \int d^{3} x d^{3} y\left(\frac{\delta^{2} \Psi}{\delta q^{a}(x) \delta q^{b}(y)}\right) \Delta q^{a}(x) \Delta q^{b}(y)+\ldots\right] \\
& =\Psi\left(q^{\prime}, \tau\right)+\left[T_{0}+T_{1}+T_{2}\right]+O\left(\epsilon^{2}\right) \\
& =U_{\epsilon} \Psi\left(q^{\prime}, \tau\right)
\end{aligned}
$$


where the $T_{n}$ represent terms with $n$ functional derivatives of $\Psi$ and one power of $\epsilon$, and

$$
\begin{aligned}
\Delta S & =-\frac{1}{\kappa} \int d^{3} x \sqrt{-\sqrt{g} U G_{a b} \Delta q^{a} \Delta q^{b}} \\
\Delta q^{a} & =q^{a}-q^{a}
\end{aligned}
$$

In order to obtain $U_{\epsilon}$, we need to evaluate

$$
\begin{aligned}
<\Delta q^{a}\left(x_{1}\right) \Delta q^{b}\left(x_{2}\right)>= & \int D(\Delta q)(\mu)_{0} \Delta q^{a}\left(x_{1}\right) \Delta q^{b}\left(x_{2}\right) \\
& \times \exp \left[-\frac{1}{\kappa} \int d^{3} x(\sqrt{g})_{0} \sqrt{-\left(\mathcal{G}_{a b}\right)_{0} \Delta q^{a} \Delta q^{b}} / \sqrt{-i \epsilon \hbar}\right](73)
\end{aligned}
$$

where $\mathcal{G}_{a b}$ is the modified supermetric

$$
\mathcal{G}_{a b} \equiv \frac{1}{\sqrt{g}} U G_{a b}
$$

and ()$_{0}$ indicates that the quantity in parenthesis is evaluated at $q=q^{\prime}$.

Clearly, $\langle\Delta q \Delta q>$ is a highly singular quantity, and is only well-defined in the context of a regularization procedure. In the absence of a non-perturbative regulator which preserves the exact diffeomorphism invariance, we work with a naive lattice regulator in which the continuous degrees of freedom labeled by $x$ are replaced by a discrete set, labeled by $n$, associated with regions of volume $v_{n}$. We have in mind, e.g., a Regge-style discretization of a continuous 4-manifold into a fixed number $N_{p}$ of simplices of varying volume. As $N_{p} \rightarrow \infty$, the choice of $v_{n}$ is of course required to be irrelevant in computing the evolution operator, as long as the regions' volumes $v_{n} \rightarrow 0$ in this limit. As we will see, this requirement is not satisfied trivially or automatically. We take the naive lattice-continuum correspondences to be

$$
\begin{aligned}
\Delta q^{a}(x) & \leftrightarrow \Delta q^{a}(n) \\
\int d^{3} x \sqrt{g} & \leftrightarrow \sum_{n=1}^{N_{p}} v_{n} \\
\frac{\delta}{\delta q^{a}(x)} & \leftrightarrow\left(\frac{\delta}{\delta q^{a}(n)}\right)_{R} \equiv \frac{\sqrt{g(n)}}{v_{n}} \frac{\partial}{\partial q^{a}(n)} \\
D q & \leftrightarrow \prod_{n} d^{D} q(n)
\end{aligned}
$$

With such a discretization, we have

$$
\begin{aligned}
<\Delta q^{a}(n) \Delta q^{b}(m)> & =\int \prod_{j} d^{D} q(j) \mu \Delta q^{a}(n) \Delta q^{b}(m) \\
& \times \exp \left[-\frac{1}{\kappa} \sum_{k} v_{k} \sqrt{-\mathcal{G}_{a b} \Delta q^{a}(k) \Delta q^{b}(k)} / \sqrt{-i \epsilon \hbar}\right]
\end{aligned}
$$


The supermetric $G_{a b}$ for the discretized degrees of freedom $\left\{q^{a}(n)\right\}$ still has Lorentzian signature, and we can follow the steps of the last section in integrating over the $q^{a}$ at each $n$. The result is

$$
<\Delta q^{a}(n) \Delta q^{b}(m)>=i \epsilon \hbar(D+1) \kappa^{2} \mathcal{G}^{a b} \frac{1}{v_{n}^{2}} \delta_{m n}
$$

and we find for the $T_{2}$ term

$$
\begin{aligned}
T_{2} & =i \epsilon \hbar \frac{(D+1)}{2} \kappa^{2} \sum_{n} \mathcal{G}^{a b} \frac{1}{v_{n}^{2}} \frac{\partial^{2}}{\partial q^{a}(n) \partial q^{b}(n)} \Psi \\
& =i \epsilon \hbar \frac{(D+1)}{2} \kappa^{2} \sum_{n} \sqrt{g} \frac{1}{U} G^{a b} \frac{1}{v_{n}^{2}} \frac{\partial^{2}}{\partial q^{a}(n) \partial q^{b}(n)} \Psi \\
& =i \epsilon \hbar \frac{(D+1)}{2} \kappa^{2} \sum_{n}\left\{\frac{1}{\sqrt{g} U} G^{a b}\left(\frac{\delta}{\delta q^{a}(n)}\right)_{R}\left(\frac{\delta}{\delta q^{b}(n)}\right)_{R} \Psi\right\}
\end{aligned}
$$

The term in braces has a simple continuum limit and, if this term were weighted by a volume factor $v_{n}$, then the continuum limit would be simply

$$
T_{2}=i \epsilon \hbar \frac{(D+1)}{2} \kappa^{2} \int d^{3} x U^{-1} G^{a b} \frac{\delta^{2}}{\delta q^{a} \delta q^{b}} \Psi \quad \text { (wrong) }
$$

There is, however, no such $v_{n}$ weighting factor in the sum, which means that the contribution of each term at each position $n$

$$
\frac{1}{\sqrt{g} U} G^{a b}\left(\frac{\delta}{\delta q^{a}(n)}\right)_{R}\left(\frac{\delta}{\delta q^{b}(n)}\right)_{R} \Psi
$$

is weighted equally, regardless of the cell or simplex volume $v_{n}$. As a consequence, even in the $v_{n} \rightarrow 0$ limit, the final answer for the state evolution would seem to depend on the distribution of volumes $\left\{v_{n}\right\}$.

Such regularization dependence never arises in ordinary quantum field theory. There may be other regularization issues, such as renormalization and anomalies, but certainly one doesn't encounter this kind of dependence on the distribution of cell volumes in computing the naive continuum limit of the Hamiltonian operator. Since the problem doesn't arise in ordinary quantum field theory, why does it come up here? The reason, of course, can be traced back to the square-root form of the action, which gives a factor of $1 / v_{n}^{2}$, rather than a factor of $1 / v_{n}$, in the correlator (77). The additional power of $1 / v_{n}$ is the source of the (apparent) difficulty. There is only one way out, if the evolution operator is not to depend on the $\left\{v_{n}\right\}$ distribution: we must impose a constraint on the physical states $\Psi$ such that the term (80) is independent of the discretized position label $n$, at least in the $v_{n} \rightarrow 0$ limit. In that case, we have

$$
T_{2}=i \epsilon \hbar \frac{(D+1) N_{p}}{2} \frac{1}{\sqrt{g} U} \kappa^{2} G^{a b}\left(\frac{\delta}{\delta q^{a}(n)}\right)_{R}\left(\frac{\delta}{\delta q^{a}(n)}\right)_{R} \Psi \quad(\text { any } n)
$$


The other terms $T_{0}$ and $T_{1}$ are operator-ordering contributions which, in the absence of an exact diffeomorphism-invariant regulator, will not be considered further here. Now absorbing the factor $\frac{1}{2}(D+1) N_{p}$ into a redefinition of $\epsilon$, and taking the continuum limit, we arrive at

$$
i \hbar \frac{1}{\sqrt{g} U} \kappa^{2} G^{a b} \frac{\delta^{2}}{\delta q^{a} \delta q^{b}} \Psi=\partial_{\tau} \Psi \quad(\text { all } x)
$$

Expanding

$$
\Psi(q, \tau)=\sum_{\mathcal{E}} a_{\mathcal{E}} e^{i \mathcal{E} \tau / \hbar} \Phi_{\mathcal{E}}(q)
$$

eq. (82) requires that for each stationary state

$$
\frac{1}{\sqrt{\mathcal{E}}} \mathcal{H}^{\mathcal{E}} \Phi_{\mathcal{E}}=\left\{-\hbar^{2} \frac{\kappa^{2}}{\mathcal{E}} G^{a b} \frac{\delta^{2}}{\delta q^{a} \delta q^{b}}+\sqrt{g} U\right\} \Phi_{\mathcal{E}}=0
$$

which is simply the Wheeler-DeWitt equation (up to operator-ordering contributions), with an effective value of Planck's constant rescaled by

$$
\hbar_{e f f}=\frac{\hbar}{\sqrt{\mathcal{E}}}
$$

Moreover, the Wheeler-DeWitt equation is consistent with, and in fact implies (via the Moncrief-Teitelboim interconnection theorem [7]), the supermomentum constraints

$$
\mathcal{H}^{i} \Phi_{\mathcal{E}}=0
$$

which are needed to compensate the gauge choice $N_{i}=0$. Thus, each stationary state $\Phi_{\mathcal{E}}$ satisfies the usual constraint algebra of general relativity, with a rescaled value of Planck's constant. The Hilbert space of all physical states is spanned by the stationary states, with all possible values of $\mathcal{E}$. Finally, multiplying both sides of (82) by $N \sqrt{g} U$, where $N$ is an arbitrary function, integrating over space, and applying the supermomentum constraint (86), we obtain the equation of motion

$$
\begin{aligned}
i \hbar \partial_{\tau} \Psi & =\left[\frac{1}{\int d^{3} x^{\prime} \sqrt{g} N U} \kappa^{2} \int d^{3} x N G^{a b}\left(-\hbar^{2} \frac{\delta^{2}}{\delta q^{a} \delta q^{b}}\right)\right] \Psi \\
& =\frac{1}{m_{P}} \int d^{3} x\left[-\hbar^{2} \tilde{N} \kappa^{2} G^{a b} \frac{\delta^{2}}{\delta q^{a} \delta q^{b}}+N_{i} \mathcal{H}_{x}^{i}\right] \Psi \\
& =Æ \Psi
\end{aligned}
$$

where

$$
Æ=\frac{1}{m_{P}} \int d^{3} x\left[-\hbar^{2} \tilde{N} \kappa^{2} G^{a b} \frac{\delta^{2}}{\delta q^{a} \delta q^{b}}+N_{i} \mathcal{H}_{x}^{i}\right]
$$


and

$$
\tilde{N}(x) \equiv m_{P} \frac{N(x)}{\int d^{3} x^{\prime} \sqrt{g} N U(q)}
$$

with $m_{P}$ an arbitrary parameter of dimension of mass.

The evolution equation (87) was obtained in ref. [5] by a transfer matrix approach, and shown to correspond to the usual classical evolution via the Ehrenfest principle. Here we have instead used the path integral to obtain a unitary evolution operator, as in the real-time Feynman approach, and avoided the signature rotation of the supermetric which was required in deriving the transfer matrix. The main point of this section is that, in performing the "real-time" path-integral of the BSW action

$$
\begin{aligned}
\Psi[ & \left.q_{f}(x), \tau_{0}+\Delta \tau\right] \\
= & \int D q(x, \tau) e^{c_{0} S[q(\tau)]} \Psi\left[q_{0}(x), \tau_{0}\right] \\
= & \lim _{\epsilon \rightarrow 0} \prod_{n=0}^{\Delta \tau / \epsilon-1} D q_{n}^{a}(x) \mu_{\epsilon}\left[q_{n}\right] \exp \left[\frac{1}{\sqrt{-i \epsilon \hbar}} \sum_{m=1}^{\Delta \tau / \epsilon-1} S\left[q_{m+1}(x), q_{m}(x)\right]\right] \\
& \times \Psi\left[q_{0}(x), \tau_{0}\right] \\
= & \lim _{\epsilon \rightarrow 0}\left(U_{\epsilon}\right)^{\Delta \tau / \epsilon} \Psi\left(q_{f}(x), \tau_{0}\right) \\
= & e^{-i \notin \Delta \tau / \hbar} \Psi\left[q_{f}(x), \tau_{0}\right]
\end{aligned}
$$

it is necessary, as in the minisuperspace case, to integrate over all possible paths, including those for which the lapse function

$$
N(x)=\left[-\frac{1}{4 \kappa^{2} \sqrt{g} U} G_{a b} \partial_{t} q^{a} \partial_{t} q^{b}\right]^{1 / 2}
$$

is imaginary. Real-valued lapse functions correspond to Lorentzian 4-manifolds, imaginary values correspond to Euclidean signature. If the paths are restricted to real $N(x)$ only, then we find that due to the Lorentzian signature of the supermetric, the integrals in eq. (76) are singular despite the regularization.

The conclusion is that in order to obtain a unitary evolution of states, we are required to sum over 4-manifolds of both Lorentzian and Euclidean signature, and in general over manifolds which may be Lorentzian in some regions, and Euclidean in others. This raises the obvious question of why spacetime seems to have Lorentzian signature, rather than Euclidean or mixed signature.

The question "why is spacetime Lorentzian?" can be raised already at the level of classical general relativity. Einstein's equations themselves do not specify a choice of metric signature; there are Lorentzian solutions to these equations, and there are Riemannian solutions. Recently, solutions to the Einstein equations in 
which part of the manifold is Riemannian (Euclidean signature) and the rest is Lorentzian have been studied [13]; it is conceivable that solutions of this kind are relevant to the very early Universe. In any case, the signature of a manifold solving the Einstein equations is determined in general from initial conditions $\left\{g_{i j}, p^{i j}\right\}$ satisfying the appropriate constraints. A given initial 3-manifold may trace out either a Lorentzian or Riemannian 4-manifold, depending on the initial choice of conjugate momenta.

The dependence of lapse on initial conditions applies also to the quantum theory. The general solution of the evolution equation (48) for the "relativistic particle" example, with $\Subset$ given in eq. (38), is

$$
\psi\left(x^{\mu}, \tau\right)=\int d^{4} p f(p) \exp \left[-\frac{i}{\hbar}\left(\frac{(D+1)}{2 m^{2}} p^{2} \tau+p_{\mu} x^{\mu}\right)\right]
$$

It is easy to see that

$$
<x^{\mu}>=<x^{\mu}>_{0}+\frac{(D+1)}{m^{2}}<p^{\mu}>\tau
$$

(recall that $\tau$ has units of action). So long as $f(p)=0$ for $p^{2}>0$, the expectation value of position follows a timelike path. We would expect the same situation in quantum gravity, for the same reason, namely, the Ehrenfest principle. If the initial "wavefunction of the Universe" $\Psi\left[q^{a}(x), \tau_{0}\right]$ has expectation values which are peaked around some (equivalence class of) configurations and momenta $\{q, p\}_{0}$, then the wavefunction tends to remain peaked in the neighborhood of a classical manifold which solves the Einstein equations for this initial data. Thus, despite the fact that

the path integral sums over Lorentzian and Euclidean manifolds, the probability density can still be sharply peaked at one or the other signature.

Obviously these remarks do not answer the question "why is spacetime Lorentzian?", but only replace it with another question about initial conditions. For an attempt to explain the preference for Lorentzian signature (in the context of non-timeparametrized theories) from an analysis of an effective "signature potential", see ref. [8].

\section{Quantum Theory in Curved Spacetime}

Associated with the problem of time in quantum gravity is a "problem of state." Let us return, for a moment, to the standard formulation of canonical quantum gravity, which in our language is a restriction to a single value of $\mathcal{E}$, and let $H$ be the Wheeler-DeWitt Hamiltonian. Suppose a physical state $\Psi$ is an eigenstate of 
an observable $Q$; this means that $Q \Psi$ must also be a physical state. But then

$$
H(Q \Psi)=[H, Q] \Psi=0
$$

which is not true, in general, unless $[H, Q]$ vanishes weakly. It is then problematic to construct physical states which are approximate eigenstates of, e.g., 3-geometry, or the position of the hands of a clock.

In this section we show how to construct physical states which are sharply peaked around a given 3-geometry and extrinsic curvature. Treating the metric degrees of freedom semiclassically, the dynamics of the other degrees of freedom approximates the standard quantum theory on a curved background. Of course, the WKB treatment can be extended to any other degrees of freedom (such as the hands of a clock) which behave more or less classically.

We recall that our path integral leads, in the end, to the following solution for the evolution of physical states:

$$
\Psi[q, \tau]=\sum_{\mathcal{E}, \alpha} c[\mathcal{E}, \alpha] \Phi_{\mathcal{E}, \alpha}[q] e^{i \mathcal{E} \tau / \hbar}
$$

where

$$
Æ \Phi_{\mathcal{E}, \alpha}=-\mathcal{E} \Phi_{\mathcal{E}, \alpha}
$$

and where the subscript $\alpha$ is meant to distinguish between different solutions of (96). As discussed above, eq. (96) is a one-parameter $(\mathcal{E})$ class of Wheeler-DeWitt equations

$$
\left[-\frac{\hbar^{2}}{\mathcal{E}} \kappa^{2} G^{a b} \frac{\delta^{2}}{\delta q^{a} \delta q^{b}}+\sqrt{g} U\right] \Phi_{\mathcal{E}, \alpha}=0
$$

each of which can be treated by WKB methods. To get the quantum-theory-incurved-spacetime limit, we follow the approach of Banks [9], treating the metric semiclassically, and expanding in powers of $\kappa^{2}$ (back-reaction of matter on metric will be ignored; it can presumably be dealt with following the approach of ref. [10]). Thus, write (96) in the form

$$
\left\{-\left[\frac{\hbar^{2}}{\mathcal{E}} \kappa^{2} G_{i j k l} \frac{\delta^{2}}{\delta g_{i j} \delta g_{k l}}+\frac{1}{\kappa^{2}} \sqrt{g} R\right]+\mathcal{H}_{m}^{\mathcal{E}}\right\} \Phi_{\mathcal{E}, \alpha}=0
$$

where $\mathcal{H}_{m}^{\mathcal{E}}$ is the Hamiltonian density for the non-gravitational fields, denoted $\phi$. We then make the WKB ansatz

$$
\Phi_{\mathcal{E}, \bar{g}}=\exp \left[i \sqrt{\mathcal{E}} S[g, \bar{g}] / \kappa^{2} \hbar\right] \rho_{V V}^{\mathcal{E}}[g] \psi_{m}^{\mathcal{E}}[\phi]
$$

where $S[g, \bar{g}]$ is Hamilton's principal function (the action of a 4-manifold solving the Einstein equations, bounded by the three manifolds with metric $\bar{g}_{i j}$ and $\left.g_{i j}\right)$; it 
satisfies the Einstein-Hamilton-Jacobi equation in both arguments

$$
\begin{aligned}
G_{i j k l} \frac{\delta S}{\delta g_{i j}} \frac{\delta S}{\delta g_{k l}}-\sqrt{g} R & =0 \\
G_{i j k l}[\bar{g}] \frac{\delta S}{\delta \bar{g}_{i j}} \frac{\delta S}{\delta \bar{g}_{k l}}-\sqrt{\bar{g}} R[\bar{g}] & =0
\end{aligned}
$$

The functional $\rho_{V V}^{\mathcal{E}}[g]$ is the Van-Vleck determinant, while $\psi_{m}^{\mathcal{E}}$ is a solution of the Tomonaga-Schwinger equation for quantum theory on a curved spacetime background

$$
i \hbar_{e f f} \frac{\delta \psi_{m}^{\mathcal{E}}}{\delta T(x ; g, \bar{g})}=\mathcal{H}_{m}^{\mathcal{E}} \psi_{m}^{\mathcal{E}}
$$

where

$$
\hbar_{e f f}=\frac{\hbar}{\sqrt{\mathcal{E}}}
$$

is the effective value of Planck's constant, and $T(x ; g, \bar{g})$ is a functional of the background spacetime defined by

$$
\frac{\delta}{\delta T}=2 G_{i j k l} \frac{\delta S}{\delta g_{i j}} \frac{\delta}{\delta g_{k l}}
$$

Up to this point, we have simply repeated the analysis of ref. 9]

However, the semiclassical approach to recovering ordinary quantum field theory, as outlined above, is subject to the following objection: Although the part of the wavefunction involving the non-gravitational fields obeys a Tomonaga-Schwinger equation, the metric $g_{i j}$, on which the "many-fingered" time parameter $T(x ; g, \bar{g})$ depends, is still a dynamical degree of freedom, and there is no physical state satisfying the Wheeler-DeWitt equation which has a probability distribution peaked at a particular 3-geometry $g_{i j}$, i.e the wavefunction is not peaked on any particular time-slice of a 4-manifold. In fact, the squared-modulus of the leading term in the WKB approach, i.e.

$$
\left|\exp \left[i \sqrt{\mathcal{E}} S[g, \bar{g}] / \kappa^{2} \hbar\right]\right|^{2}=1
$$

has no dependence on $g_{i j}$ at all. The best one can do in the standard formulation (that is, using only a single value of $\mathcal{E}$ ) is to superimpose WKB solutions

$$
\begin{aligned}
\Phi_{\mathcal{E}, F}[g] & =\int D \bar{g}_{i j} F\left[\bar{g}_{i j}\right] \Phi_{\mathcal{E}, \bar{g}} \\
& \left.=\int D \bar{g}_{i j} f\left[\bar{g}_{i j}\right] \exp \left[i\left\{\sqrt{\mathcal{E}} S[g, \bar{g}] / \kappa^{2}+\theta[\bar{g}]\right\} / \hbar\right\}\right] \rho_{V V}^{\mathcal{E}} \psi_{m}^{\mathcal{E}}
\end{aligned}
$$


where $f[\bar{g}]$ is a real functional peaked (modulo diffeomorphisms) at a particular 3-geometry $g_{0 i j}$, and we define

$$
p_{0}^{i j} \equiv\left(\frac{\delta \theta}{\delta \bar{g}_{i j}}\right)_{\mid \bar{g}=g_{0}}
$$

where $\theta[\bar{g}]$ is the phase of the smearing functional $F[\bar{g}]$. As shown many years ago by Gerlach [11, this superposition is still not peaked at any one 3-geometry, but rather on all three-geometries which are spacelike slices of a certain 4-manifold, satisfying Einstein's equations with initial data $\left\{g_{0 i j}, p_{0}^{i j}\right\}$. Thus there is no physical state, and no subspace of physical states, which would correspond to an eigenstate of a non-stationary observable (such as the three-geometry, or the fields on a given three-geometry).

It is at this point that we make use of the freedom, inherent in our formulation, to superimpose states of different $\mathcal{E}$, and write

$$
\begin{aligned}
\Psi\left[g_{i j}, \phi, \tau\right] & =\int d \mathcal{E} D \bar{g} F[\bar{g}, \mathcal{E}] \exp \left[\frac{i}{\hbar}\left\{\mathcal{E} \tau+\sqrt{\mathcal{E}} S[g, \bar{g}] / \kappa^{2}\right\}\right] \rho_{V V}^{\mathcal{E}} \psi_{m}^{\mathcal{E}}[\phi, T(x ; g, \bar{g})] \\
& \approx \psi_{m}^{\mathcal{E}_{0}}\left[\phi, T\left(x ; g, g_{0}\right)\right] \int d \mathcal{E} D \bar{g} F[\bar{g}, \mathcal{E}] \exp \left[\frac{i}{\hbar}\left\{\mathcal{E} \tau+\sqrt{\mathcal{E}} S[g, \bar{g}] / \kappa^{2}\right\}\right] \rho_{V V}^{\mathcal{E}} \\
& =\psi_{m}^{\mathcal{E}_{0}}\left[\phi, T\left(x ; g, g_{0}\right)\right] \Phi_{F}^{\left(g_{0}, p_{0}\right)}\left[g_{i j}, \tau\right]
\end{aligned}
$$

where it is assumed that $F[\bar{g}, \mathcal{E}]$ is sharply peaked around $\mathcal{E}=\mathcal{E}_{0}, \bar{g}_{i j}=g_{0 i j}$, and $p_{0}^{i j}$ is defined as in eq. (106) above. The $\psi_{m}$ factor can be pulled outside the integral, on the grounds that its variation with $\mathcal{E}$ and $\bar{g}$ is much less than that of the smearing function $F[\bar{g}, \mathcal{E}]$, and the $\exp \left[i \sqrt{\mathcal{E}} S / \kappa^{2} \hbar\right]$ factor. Now consider the leading WKB term

$$
\Phi_{F}^{\left(g_{0}, p_{0}\right)}\left[g_{i j}, \tau\right]=\int d \mathcal{E} D \bar{g}_{i j} f[\mathcal{E}, \bar{g}] \exp \left[\frac{i}{\hbar}\left\{\mathcal{E} \tau+\sqrt{\mathcal{E}} S[g, \bar{g}] / \kappa^{2}+\theta[\bar{g}]\right\}\right] \rho_{V V}^{\mathcal{E}}
$$

This wavefunction will be peaked at configurations $g_{i j}$ where the phase in the integrand is stationary, with respect to small variations in $\bar{g}_{i j}$ and $\mathcal{E}$ around $g_{0 i j}$ and $\mathcal{E}_{0}$, respectively. In other words, the wavefunction is peaked at metrics $g_{i j}$, at time $\tau$, such that

$$
\begin{aligned}
\tau & =-\frac{1}{2 \kappa^{2} \sqrt{\mathcal{E}}_{0}} S\left[g, g_{0}\right] \\
p_{0}^{i j} & =-\frac{\sqrt{\mathcal{E}_{0}}}{\kappa^{2}}\left(\frac{\delta S[g, \bar{g}]}{\delta \bar{g}_{i j}}\right)_{\mid \bar{g}=g_{0}}
\end{aligned}
$$

The second of these two equations is satisfied by the metric $g_{i j}$ of any time-slice of a 4-manifold, satisfying Einstein's equations with initial data $\left\{g_{0 i j}, p_{0}^{i j}\right\}$. The 
first equation requires that the action of the 4-manifold between the initial slice $g_{0 i j}$ and the given slice $g_{i j}$ is proportional to the time-parameter $\tau$. Now consider a foliation of the given 4-manifold parametrized by some variable $x_{0}$, with $g_{i j}=g_{0 i j}$ at $x_{0}=0$. Hamilton's principal function $S\left[g, g_{0}\right]$ is monotonic in $x_{0}$, which means that $S\left[g, g_{0}\right]=0$ only for $g_{i j}=g_{0 i j}$. It follows that, at $\tau=0$, eq. (109) gives us

$$
0=S\left[g, g_{0}\right] \quad \Longrightarrow \quad g_{i j}=g_{0 i j} \quad \text { (modulo diffeomorphisms) }
$$

As a consequence, at $\tau=0$, the wavefunction $\Phi_{F}^{\left(g_{0}, p_{0}\right)}[g, \tau=0]$ is peaked at $g_{i j}=g_{0 i j}$ (modulo diffeomorphisms). Thus, from the definition of the many-fingered time variable, where $T\left(x ; g_{0}, g_{0}\right)=0$,

$$
\begin{aligned}
\Psi\left[g_{i j}, \phi, \tau=0\right] & =\Phi_{F}^{\left(g_{0}, p_{0}\right)}[g, \tau=0] \times \psi_{m}^{\mathcal{E}_{0}}\left[\phi, T\left(x ; g, g_{0}\right)\right] \\
& \approx \Phi_{F}^{\left(g_{0}, p_{0}\right)}[g, \tau=0] \times \psi_{m}^{\mathcal{E}_{0}}[\phi, T=0]
\end{aligned}
$$

The importance of eq. (111) is that there exists, in our formulation, a class of states where the metric (and extrinsic curvature) is sharply peaked around a given geometry $g_{0 i j}\left(\right.$ and $p_{0}^{i j}$ ), and where the state factorizes into a wavefunction $\left(\Phi_{F}\right)$ suppressing fluctuations away from the given 3-geometry, and a wavefunction $\left(\psi_{m}\right)$ describing the state of the non-gravitational fields on that 3-geometry. Such states can be fairly described as eigenstates of non-stationary observables; these eigenstates are impossible to construct, as physical states, in the standard formulation of canonical quantum gravity.

Finally, we consider transition probabilities. Take an initial state of the form

$$
\Psi_{i n}\left[g_{i j}, \phi\right]=\Phi_{F}^{\left(g_{0}, p_{0}\right)}[g, 0] \times \psi_{m}^{\mathcal{E}_{0}}[\phi, 0]
$$

and a final state of similar form

$$
\Psi_{f}\left[g_{i j}, \phi\right]=\Phi_{F^{\prime}}^{\left(g_{0}^{\prime}, p_{0}^{\prime}\right)}[g, 0] \times \psi_{m}^{\prime \mathcal{E}_{0}}[\phi, 0]
$$

where the smearing function $F^{\prime}$ is peaked around some time slice $\left(g_{0}^{\prime}, p_{0}^{\prime}\right)$ of the classical 4-geometry specified by the initial data $\left(g_{0}, p_{0}\right)$. The transition probability for $\Psi_{i n} \rightarrow \Psi_{f}$ after a time $\tau$ is given by the factorized expression

$$
\begin{aligned}
P_{i n \rightarrow f}(\tau) & =\left|<\Psi_{f}\right| e^{-i Æ \tau / \hbar}\left|\Psi_{i n}>\right|^{2} \\
& =\left|<\Psi_{f}\right| \Psi_{i n}(\tau)>\left.\right|^{2} \\
& =\left|<\Phi_{F^{\prime}}^{\left(g_{0}^{\prime}, p_{0}^{\prime}\right)}[g, 0]\right| \Phi_{F}^{\left(g_{0}, p_{0}\right)}[g, \tau]>\left.\right|^{2} \\
& \times\left|<\psi_{m}^{\prime \mathcal{E}_{0}}[\phi, 0]\right| \psi_{m}^{\mathcal{E}_{0}}\left[\phi, T\left(x ; g_{0}^{\prime}, g_{0}\right)\right]>\left.\right|^{2}
\end{aligned}
$$


The first of these factors

$$
\left|<\Phi_{F^{\prime}}^{\left(g_{0}^{\prime}, p_{0}^{\prime}\right)}[g, 0]\right| \Phi_{F}^{\left(g_{0}, p_{0}\right)}[g, \tau]>\left.\right|^{2}
$$

gives the probability, after a time $\tau$, to be on the time-slice described by $\left(g_{0}^{\prime}, p_{0}^{\prime}\right)$ (up to a certain uncertainty, specified by the smearing function $F^{\prime}$ ). The second factor

$$
\left|<\psi_{m}^{\prime \mathcal{E}_{0}}[\phi, 0]\right| \psi_{m}^{\mathcal{E}_{0}}\left[\phi, T\left(x ; g_{0}^{\prime}, g_{0}\right)\right]>\left.\right|^{2}
$$

is the quantum-field-theory-in-curved-spacetime result; it gives the probability for a transition from an initial state $\psi_{m}$ of quantum fields on the time-slice $g_{0}$, to the state $\psi_{m}^{\prime}$ on the later time-slice $g_{0}^{\prime}$. Both the initial and final 3-manifolds are timeslices of the same 4-manifold specified by the initial data $\left\{g_{0}, p_{0}\right\}$, and the state $\psi_{m}[\phi, T]$ evolves according to the Tomonaga-Schwinger equation (101).

In this way, we see how approximate eigenstates of geometry and extrinsic curvature may be constructed, and how the standard formalism of quantum field theory in curved spacetime emerges. We will not attempt to go further and discuss the problem of measurement in this context, apart from noting that any of the standard "realistic" approaches that have been applied to non-relativistic quantum mechanics, e.g. many-universes, decoherence, or Bohm's theory, can be applied in our formulation as well.

\section{Inclusion of Fermions}

We have so far assumed that the canonical momenta $p_{a}$ appear quadratically in the Hamiltonian, with indices contracted by the supermetric. The Hamiltonian of a set of Dirac fields, on the other hand, is linear in the fermionic momenta, and it is not immediately obvious how such fields are incorporated into our approach.

In our previous work [5] we found two independent methods for determining the $Æ$ operator. The "undetermined constant" method was based on the trivial observation that the actions $S$ and $S^{\prime}=$ const. $\times S$ are equivalent at the classical level; this leads to the fact that the ratio of the kinetic and potential terms of the Hamiltonian (which is the $\rightleftarrows$ functional), is indeterminate at the classical level. The second method, leading to the same quantum theory, is the transfer matrix method, whose "real-time" or Feynman version was presented in the preceding sections. We will now apply both methods to obtain the $Æ$ operator for gravity coupled to a Dirac field.

The action for the Einstein-Dirac system is expressed in terms of the fermion field $\psi$ and tetrad field $e^{a}{ }_{\mu}$ as

$$
S_{E D}=\int d^{4} x \operatorname{det}(e)\left[{ }^{4} R+i \bar{\psi}\left(e_{a}^{\mu} \gamma^{a} D_{\mu}-m\right) \psi\right]
$$


where $D_{\mu}$ is the usual covariant spinor derivative. The extension of the canonical ADM formalism to this system was worked out in ref. [12], for the "time-gauge"

$$
e_{i}^{0}=0 \quad(i=1,2,3)
$$

In this gauge, the Einstein-Dirac action expressed in terms of canonical momenta has the form

$$
S=\int d^{4} x\left[p_{a} \dot{q}^{a}+\pi_{\psi} \dot{\psi}-\left(N \mathcal{H}+N_{i} \mathcal{H}^{i}+\epsilon^{i j} M_{i j}\right)\right]
$$

where the $q^{a}$ are the triad fields $e_{k}^{c}(x)$, and

$$
\begin{aligned}
\mathcal{H} & =\kappa^{2} G^{a b} p_{a} p_{b}+\sqrt{g} U+\mathcal{H}_{\psi} \\
\mathcal{H}_{\psi} & =\pi_{\psi} K \psi=\pi_{\psi} \gamma^{0}\left[e_{a}^{i} \gamma^{a} D_{i}-m\right] \psi
\end{aligned}
$$

The first-class constraints are

$$
\mathcal{H}=\mathcal{H}^{i}=M_{i j}=0
$$

where the supermomenta $\mathcal{H}^{i}$ and the generators of local frame rotations $M_{i j}$ are linear in the momenta. In addition there are 2nd-class constraints, some of which are associated with the time-gauge condition (118), and also which relate $\pi_{\psi}$ to $\bar{\psi}$ :

$$
\pi_{\psi}=i \sqrt{g} \bar{\psi} \gamma^{0}
$$

The 2nd-class constraints are handled, according to the Dirac procedure, by replacing Poisson brackets by Dirac brackets. The explicit form of all constraints in terms of the canonical variables, and other details, may be found in ref. [12].

Now consider an alternative action $S_{E D}^{\prime}$ which differs from $S_{E D}$ only by a multiplicative constant

$$
S_{E D}^{\prime}=\sqrt{\mathcal{E}} S_{E D}
$$

Obviously, the equations of motion derived from $S_{E D}^{\prime}$ are identical to those derived from $S_{E D}$. The constant $\mathcal{E}$ is therefore irrelevant at the classical level. In going to the canonical formulation, however, we find that

$$
\begin{aligned}
S^{\prime} & =\int d^{4} x\left[p_{a} \dot{q}^{a}+\pi_{\psi} \dot{\psi}-\left(N \mathcal{H}^{\mathcal{E}}+N_{i} \mathcal{H}^{i}+\epsilon^{i j} M_{i j}\right]\right. \\
\mathcal{H}^{\mathcal{E}} & =\frac{1}{\sqrt{\mathcal{E}}} \kappa^{2} G^{a b} p_{a} p_{b}+\mathcal{H}_{\psi}+\sqrt{\mathcal{E}} \sqrt{g} U
\end{aligned}
$$

with 2nd class constraints enforcing

$$
\pi_{\psi}=i \sqrt{\mathcal{E}} \sqrt{g} \bar{\psi} \gamma^{0}
$$

\footnotetext{
${ }^{1}$ We take right derivatives with respect to $\psi$.
} 
Define

$$
H^{\mathcal{E}} \equiv \int d^{3} x\left(N \mathcal{H}^{\mathcal{E}}+N_{i} \mathcal{H}^{i}+\epsilon^{i j} M_{i j}\right)
$$

and consider a field configuration $\left\{e_{i}^{a}(x, t), \psi(x, t), \bar{\psi}(x, t)\right\}$ which solves the Hamiltonian equations of motion derived from $H^{\mathcal{E}}$, for some given value of $\mathcal{E}$. Then it is clear that this configuration is a solution for any other value of $\mathcal{E}$, since the classical orbits in configuration space (i.e. solutions of the Euler-Lagrange equations) are independent of $\mathcal{E}$. In general then, the Dirac bracket equation of motion

$$
\partial_{t} F=\left\{F, H^{\mathcal{E}}\right\}_{D}
$$

supplemented by the first class constraints

$$
\begin{aligned}
H^{\mathcal{E}} & =0 \quad \text { for any } N, N_{i}, \epsilon^{i j} \\
\Longrightarrow \mathcal{H}^{\mathcal{E}} & =\mathcal{H}^{i}=M_{i j}=0
\end{aligned}
$$

generates a set of orbits in configuration space which is independent of $\mathcal{E}$. In this sense $\mathcal{E}$ is "classically irrelevant."

Now observe that the constraint $H^{\mathcal{E}}=0$ can be written

$$
\rightleftarrows=-\mathcal{E} \quad \text { for any } N, N_{i}, \epsilon^{i j}
$$

where $Æ$ is defined implicitly by

$$
\rightleftarrows=\int d^{3} x\left\{\frac{N\left(\kappa^{2} G^{a b} p_{a} p_{b}+\sqrt{-Æ} \mathcal{H}_{\psi}\right)}{\int d^{3} x^{\prime} \sqrt{g} N U}+\frac{1}{m_{p}}\left(N_{i} \mathcal{H}^{i}+\epsilon^{i j} M_{i j}\right)\right\}
$$

and where $m_{p}$ is an arbitrary parameter with dimensions of mass. From this definition, it is straightforward to show that, for any functional $F=F\left[q, p, \psi, \pi_{\psi}\right]$, the Poisson bracket with $Æ$ is related to the corresponding Poisson bracket with $H^{\mathcal{E}}$ via

$$
\begin{aligned}
\left.m_{p}\{F, \circledast\}\right\} & \int d^{3} x\left\{\left[1+\frac{\int d^{3} x_{1} N \mathcal{H}_{\psi}}{2 \sqrt{\mathcal{E}} \int d^{3} x_{2} N \sqrt{g} U}\right]^{-1} \frac{\sqrt{\mathcal{E}} m_{p} N}{\int d^{3} x_{3} N \sqrt{g} U}\left\{F, \mathcal{H}^{\mathcal{E}}\right\}\right. \\
& \left.+N_{i}\left\{F, \mathcal{H}^{i}\right\}+\epsilon^{i j}\left\{F, M_{i j}\right\}\right\} \\
= & \left\{F, H^{\mathcal{E}}\right\}^{N \rightarrow \tilde{N}}
\end{aligned}
$$

where

$$
\tilde{N}(N) \equiv\left[1+\frac{\int d^{3} x_{1} N \mathcal{H}_{\psi}}{2 \sqrt{\mathcal{E}} \int d^{3} x_{2} N \sqrt{g} U}\right]^{-1} \frac{\sqrt{\mathcal{E}} m_{p} N}{\int d^{3} x_{3} N \sqrt{g} U}
$$

\footnotetext{
${ }^{2}$ Of course, $\left\{F, H^{\mathcal{E}}\right\}_{D} \approx\left\{F, H^{\mathcal{E}}\right\}$, since $\mathcal{H}^{\mathcal{E}}=0$ is a first-class constraint.
} 
Eq. (130) is derived by simply carrying out the functional derivatives contained in the Poisson brackets shown, and applying the constraint (128). Then, since the Dirac bracket $\{F, \mathbb{E}\}_{D}$ is linear in Poisson brackets $\{. ., \mathbb{E}\}$, eq. (130) implies

$$
m_{p}\{F, \circledast\}_{D}=\left\{F, H^{\mathcal{E}}\right\}_{D}^{N \rightarrow \tilde{N}}
$$

Defining $\tau=m_{p} t$, this demonstrates the equivalence of

$$
\partial_{t} O=\left\{O, H^{\mathcal{E}}\right\}_{D} \quad \Leftrightarrow \quad \partial_{\tau} O=\{O, \mathbb{E}\}_{D}
$$

up to a time-reparametrization, expressed by $N \rightarrow \tilde{N}$. Note that $N(x)$ and const. $\times$ $N(x)$ have the same $\tilde{N}$.

We now quantize by replacing Dirac brackets with commutators (in the case of bosonic fields), and anticommutators (in the case of fermionic fields). Time evolution of states is given by the Schrödinger equation

$$
i \hbar \partial_{\tau} \Psi[q, \tilde{\psi}, \tau]=\circledast \Psi[q, \tilde{\psi}, \tau]
$$

with the general solution

$$
\Psi[q, \tilde{\psi}, \tau]=\sum_{\mathcal{E}} a_{\mathcal{E}} \Phi_{\mathcal{E}}[q, \tilde{\psi}] e^{i \mathcal{E} \tau / \hbar}
$$

where

$$
\tilde{\psi} \equiv g^{\frac{1}{4}} \psi
$$

and $\Phi_{\mathcal{E}}$ satisfies a Wheeler-DeWitt equation

$$
\frac{1}{\sqrt{\mathcal{E}}} \mathcal{H}^{\mathcal{E}} \Phi_{\mathcal{E}}=\left[-\frac{\hbar^{2}}{\mathcal{E}} \kappa^{2} " G^{a b} \frac{\delta^{2}}{\delta q^{a} \delta q^{b}} "+i \frac{\hbar}{\sqrt{\mathcal{E}}} \frac{\delta}{\delta \tilde{\psi}} K \tilde{\psi}+\sqrt{g} U\right] \Phi_{\mathcal{E}}=0
$$

where the operator-ordering remains to be specified. Note that, as in the purely bosonic case, the classically irrelevant constant $\mathcal{E}$ can be absorbed into a redefinition of $\hbar$

$$
\hbar_{e f f}=\frac{\hbar}{\sqrt{\mathcal{E}}}
$$

This concludes the first, "undetermined constant" method for finding the $Æ$ operator.

Next we apply the path-integral approach, following as closely as possible the procedure of the previous section for the purely bosonic case. Since the generalized BSW action for gravity + fermions will contain a factor of $\mathcal{H}_{\psi}$ inside the square-root, our strategy will be to expand the path-integrand in powers of $\mathcal{H}_{\psi}$, and evaluate the relevant expressions to some finite order (first order, in this article). These expressions can then be compared, order by order in $\mathcal{H}_{\psi}$, with results of the "undetermined constant" method above. 
We again set $N_{i}=0$, and also $\epsilon^{i j}=0$, which is to be compensated by imposing the corresponding physical state constraints

$$
\mathcal{H}^{i} \Psi=0 \quad M_{i j} \Psi=0
$$

Solving for the bosonic momenta in terms of the time-derivatives

$$
p_{a}=\frac{1}{2 \kappa^{2} N} G_{a b} \partial_{t} q^{b}
$$

inserting into the Hamiltonian constraint

$$
\frac{1}{4 \kappa^{2} N^{2}} G_{a b} \partial_{t} q^{a} \partial_{t} q^{b}+\sqrt{g} U+\mathcal{H}_{\psi}=0
$$

and solving for the lapse function

$$
N=\frac{1}{2 \kappa}\left[-\frac{1}{\left(\sqrt{g} U+\mathcal{H}_{\psi}\right)} G_{a b} \partial q^{a} \partial q^{b}\right]^{1 / 2}
$$

we arrive at a square-root action

$$
S=\int d^{4} x\left\{i \sqrt{g} \bar{\psi} \gamma^{0} \partial_{t} \psi-\frac{1}{\kappa} \sqrt{-\left(\sqrt{g} U+\mathcal{H}_{\psi}\right) G_{a b} \partial_{t} q^{a} \partial_{t} q^{b}}\right\}
$$

The corresponding path-integral is

$$
\begin{aligned}
\Psi\left[q^{\prime}, \tilde{\psi}^{\prime}, \tau+\Delta \tau\right]= & \int D q D \bar{\psi} D \psi \mu(q, \psi, \bar{\psi}) \exp \left[c _ { 0 } \int d ^ { 4 } x \left\{i \sqrt{g} \bar{\psi} \gamma^{0} \partial_{t} \psi\right.\right. \\
& \left.\left.-\frac{1}{\kappa} \sqrt{-\left(\sqrt{g} U+\mathcal{H}_{\psi}\right) G_{a b} \partial_{t} q^{a} \partial_{t} q^{b}}\right\}\right] \Psi[q, \tilde{\psi}, \tau]
\end{aligned}
$$

where

$$
\mathcal{H}_{\psi}=i \sqrt{g} \bar{\psi} \gamma^{0} K \psi
$$

and $c_{0}$ represents the $\epsilon \rightarrow 0$ continuum limit of the regularization-dependent constant $c_{\epsilon} \propto 1 / \sqrt{-i \epsilon \hbar}$. Now expand the exponential to first order in $\mathcal{H}_{\psi}$

$$
\begin{aligned}
& \Psi\left[q^{\prime}, \tilde{\psi}^{\prime}, \tau+\Delta \tau\right] \\
& =\quad \int D q D \bar{\psi} D \psi \mu(q, \psi, \bar{\psi}) \\
& \quad \times\left[1-\frac{c_{0}}{\kappa} \int d^{3} x d \tau \frac{\mathcal{H}_{\psi}}{2 \sqrt{g} U} \sqrt{-\sqrt{g} U G_{a b} \partial_{\tau} q^{a} \partial_{\tau} q^{b}}\right] \\
& \quad \times \exp \left[c_{0}\left(S_{0}+\int d^{3} x d \tau i \sqrt{g} \bar{\psi} \gamma^{0} \partial_{\tau} \psi\right)\right] \Psi[q, \tilde{\psi}, \tau]
\end{aligned}
$$

where $S_{0}$ is the bosonic action

$$
S_{0}=-\frac{1}{\kappa} \int d^{3} x d \tau \sqrt{-\sqrt{g} U G_{a b} \partial_{\tau} q^{a} \partial_{\tau} q^{b}}
$$


We now regularize the path-integral according to the lattice prescription (75). The bosonic part of this path-integral, based on the action $S_{0}$, leads to the operator evolution

$$
i \hbar \partial_{\tau} \Psi=A \Psi
$$

where

$$
\begin{aligned}
A & =\frac{(D+1)}{2} \sum_{n} \frac{\sqrt{g}}{U} \kappa^{2} G^{a b} \frac{1}{v_{n}^{2}}\left(-\hbar^{2}\right) \frac{\partial^{2}}{\partial q^{a}(n) \partial q^{b}(n)} \\
& =\frac{(D+1)}{2} \sum_{n} \frac{\sqrt{g}}{U} \kappa^{2} \frac{1}{v_{n}^{2}} G^{a b} p_{a}^{L}(n) p_{b}^{L}(n)
\end{aligned}
$$

and we have defined

$$
p_{a}^{L}(n) \equiv-i \hbar \frac{\partial}{\partial q^{a}(n)}
$$

Then to zeroth-order in $\mathcal{H}_{\psi}$, we can identify the derivatives $\partial_{\tau} q$ in the term proportional to $\mathcal{H}_{\psi}$ as proportional to the bosonic momentum operators, according to

$$
\begin{aligned}
\partial_{\tau} q^{a}(n) & =\frac{\partial A}{\partial p_{a}^{L}(n)} \\
& =(D+1) \kappa^{2} \frac{\sqrt{g}}{U v_{n}^{2}} G^{a b} p_{b}^{L}(n) \\
& =\frac{(D+1) \kappa^{2}}{U v_{n}} G^{a b} p_{b}(n)
\end{aligned}
$$

where we have introduced

$$
p_{a}(n) \equiv \frac{\sqrt{g}}{v_{n}} p_{a}^{L}(n)
$$

so that, as operators, using eq. (150),

$$
p_{a}(n) \rightarrow-i \hbar\left(\frac{\delta}{\delta q^{a}(n)}\right)_{R}
$$

The regularized path-integral, to first-order in $\mathcal{H}_{\psi}$ is now

$$
\begin{aligned}
\Psi\left[q^{\prime}, \tilde{\psi}^{\prime}, \tau+\epsilon\right]= & \int D q D \bar{\psi} D \psi \mu_{\epsilon}(q)(1-\epsilon W) \exp \left[-c_{\epsilon} \sum_{n} v_{n}\left\{i \bar{\psi} \gamma^{0} \Delta \psi\right.\right. \\
& \left.\left.+\frac{1}{\kappa} \sqrt{-\mathcal{G}_{a b} \Delta q^{a} \Delta q^{b}}\right\}\right] \Psi\left[q^{\prime}+\Delta q, \tilde{\psi}^{\prime}+\Delta \tilde{\psi}, \tau\right]
\end{aligned}
$$

where

$$
W=c_{\epsilon} \frac{(D+1)}{2} \sum_{n} \frac{1}{\sqrt{g} U} \sqrt{\frac{-\kappa^{2} G^{a b} p_{a} p_{b}}{\sqrt{g} U}}\left[i \sqrt{g} \bar{\psi} \gamma^{0} K\left(\psi^{\prime}+\Delta \psi\right)\right]
$$


Carrying out the integrals over $\Delta q, \Delta \psi, \bar{\psi}$, we find

$$
\begin{aligned}
\Psi_{\tau+\epsilon}= & {\left[1+\epsilon \frac{(D+1)}{2} \sum_{n}\left\{\frac{1}{i \hbar} \frac{1}{\sqrt{g} U} \kappa^{2} G^{a b} p_{a} p_{b}\right.\right.} \\
& \left.\left.+\frac{1}{\sqrt{g} U} \sqrt{\frac{-\kappa^{2} G^{a b} p_{a} p_{b}}{\sqrt{g} U}}\left(\frac{\delta}{\delta \tilde{\psi}}\right)_{R} K \tilde{\psi}\right\}\right] \Psi_{\tau}
\end{aligned}
$$

where the square-root operator is defined via spectral analysis.

Once again, the requirement that the state evolution is independent, in the continuum limit, of the choice of $\left\{v_{n}\right\}$, implies that the term in braces is the same in each cell (simplex) $n$. Therefore, in the continuum limit,

$$
i \hbar \partial_{\tau} \Psi=\frac{1}{\sqrt{g} U}\left[\kappa^{2} G^{a b} p_{a} p_{b}+\sqrt{\frac{-\kappa^{2} G^{a b} p_{a} p_{b}}{\sqrt{g} U}} i \hbar \frac{\delta}{\delta \tilde{\psi}} K \tilde{\psi}\right] \Psi \quad(\text { all } x)
$$

where the divergent factor $N_{p}(D+1) / 2$ has been absorbed into a rescaling of $\tau$. For stationary states $\Phi_{\mathcal{E}}$ we have

$$
\left[\kappa^{2} G^{a b} p_{a} p_{b}+\sqrt{\frac{-\kappa^{2} G^{a b} p_{a} p_{b}}{\sqrt{g} U}} i \hbar \frac{\delta}{\delta \tilde{\psi}} K \tilde{\psi}+\mathcal{E} \sqrt{g} U\right] \Phi_{\mathcal{E}}=0
$$

Then, since

$$
\sqrt{\frac{-\kappa^{2} G^{a b} p_{a} p_{b}}{\sqrt{g} U}} \Phi_{\mathcal{E}}=\sqrt{\mathcal{E}} \Phi_{\mathcal{E}}+\text { order } \mathcal{H}_{\psi} \text { corrections }
$$

it follows that, up to first order in $\mathcal{H}_{\psi}$, we recover the same stationary state equation

$$
\left[-\frac{\hbar^{2}}{\mathcal{E}} \kappa^{2 "} G^{a b} \frac{\delta^{2}}{\delta q^{a} \delta q^{b}} "+i \frac{\hbar}{\sqrt{\mathcal{E}}} \frac{\delta}{\delta \tilde{\psi}} K \tilde{\psi}+\sqrt{g} U\right] \Phi_{\mathcal{E}}=0
$$

that was obtained (eq. (137)) from the "undetermined constant" approach. As in the purely bosonic case, the imposition of $\mathcal{H}^{\mathcal{E}} \Phi_{\mathcal{E}}=0$ for all $\mathcal{E}$ at every point $x$ is consistent with, and in fact implies (c.f. ref. [0]), the other required first-class constraints $\mathcal{H}^{i} \Psi=M_{i j} \Psi=0$, up to the usual operator-ordering issues.

\section{Conclusions}

For ordinary quantum theories without time-parametrization, the regularized path integral is expressed as a product of integrals, each of which evolves the state function unitarily over a very small time interval. In this article we have examined whether such a construction can be applied to theories with square-root, timereparametrization invariant actions. Our result is that unitarity requires: i) an 
unconventional phase in the path-integrand; and ii) summation over configurations of both real and imaginary proper-time lapse. In the case of quantum gravity, the second requirement means that path-integration must run over manifolds of Lorentzian, Euclidean and, in general, mixed signature. We have also shown how the formalism extends to fermionic actions.

Unitarity, of course, refers to evolution in a certain time parameter. In our formulation, the time parameter is simply a measure of the number of integrations in the (regulated) path-integral, evolving an initial state to a later state. This "quantum time" parameter is neither a geometrical quantity (such as a proper time lapse), nor a dynamical variable (such as the extrinsic curvature). It is, instead, a parameter which is intrinsic to the path-integral measure. The connection to classical dynamics is established via an Ehrenfest principle.

In the standard canonical formulation of quantum gravity, the physical states are solutions of a Wheeler DeWitt equation $\mathcal{H} \Psi=0$. In contrast, an outcome of our formulation is that physical states belong to a Hilbert space which is spanned by the solutions of a family of Wheeler-DeWitt equations $\mathcal{H}^{\mathcal{E}} \Phi_{\mathcal{E}}=0$, which are distin-

guished by having different effective values of Planck's constant $\hbar_{e f f}=\hbar / \sqrt{\mathcal{E}}$. As discussed in section 4 , a superposition of states with varying $\mathcal{E}$ (or $\hbar_{e f f}$ ) allows us to construct, at the semiclassical level, physical states whose amplitudes are peaked at particular 3-geometries and extrinsic curvatures. The width of the peak, in superspace, is inversely proportional to the dispersion $\Delta \mathcal{E}$. Projection operators formed from such states, and linear combinations of those projection operators, belong to the physical observables of the theory. It is worth noting that the stationary states (i.e. solutions of a Wheeler-DeWitt equation with a fixed value of $\mathcal{E}$ ) can never be peaked around any one 3-geometry. At best, in the WKB limit, a stationary state is peaked at every possible spacelike slice of some 4-manifold satisfying the Einstein equations.

If our view is correct, then the phenomenological value of Planck's constant is the mean value of a dynamical quantity, having a finite uncertainty of quantum origin. How large this uncertainty might be, and whether there could conceivably be testable consequences, are interesting issues for further investigation.

\section{Acknowledgements}

We would like to thank Maurizio Martellini for some very stimulating discussions. J.G. is also happy to acknowledge the hospitality of the Lawrence Berkeley Laboratory, and the Niels Bohr Institute. J.G.'s research is supported in part by 
the U.S. Dept. of Energy, under Grant No. DE-FG03-92ER40711. A.C.'s research is supported by an EEC fellowship in the 'Human Capital and Mobility' program, under contract No. ERBCHBICT930313. 


\section{References}

[1] K. Kuchar, in Quantum Gravity 2: A second Oxford symposium, ed. C. Isham, R. Penrose, and D. Sciama (Oxford University Press, Oxford, 1981).

[2] K. Kuchar, Time and interpretations of quantum gravity, Proceedings of the 4th Canadian Conference on General Relativity and Astrophysics, ed. G. Kunstatter et. al. (World Scientific, Singapore, 1992).

[3] C. J. Isham, Imperial College preprint IMPERIAL-TP-91-92-25, archive: gr-qc/9210011.

[4] J. Greensite, Phys. Rev. D49 (1994) 930.

[5] A. Carlini and J. Greensite, Niels Bohr Institute preprint NBI-HE-94-31, archive: gr-qc/9406044.

[6] R.F. Baierlein, D.H. Sharp, and J.A. Wheeler, Phys. Rev. 126 (1962) 1864;

J. A. Wheeler, in Relativity, Groups, and Topology, ed. C. DeWitt and B. DeWitt (Gordon and Breach, New York, 1964).

[7] V. Moncrief and C. Teitelboim, Phys. Rev. D6 (1972) 966.

[8] A. Carlini and J. Greensite, Phys. Rev. D49 (1993) 866;

J. Greensite, Phys. Lett. B300 (1993) 34.

[9] T. Banks, Nucl. Phys. B249 (1985) 332.

[10] R. Brout and G. Venturi, Phys. Rev. D39 (1989) 2436.

[11] U. Gerlach, Phys. Rev. 177 (1969) 1929.

[12] J.E. Nelson and C. Teitelboim, Ann. Phys. 116 (1978) 86.

[13] G.F.R. Ellis, A. Sumeruk, D. Coule and C. Hellaby, Class. Quantum Grav. 9 (1992) 1535;

T. Dereli and R. Tucker, Class. Quantum Grav. 10 (1993) 365;

T. Dray, C. Manogue and R. Tucker, Phys. Rev. D48, (1993) 2587;

S.A. Hayward, Class. Quantum Grav. 9 (1992) 1851. 